Daniel Aubram

\title{
Explicitly coupled consolidation analysis using piecewise constant pressure
}

Journal article | Accepted manuscript (Postprint)

This version is available at https://doi.org/10.14279/depositonce-9145

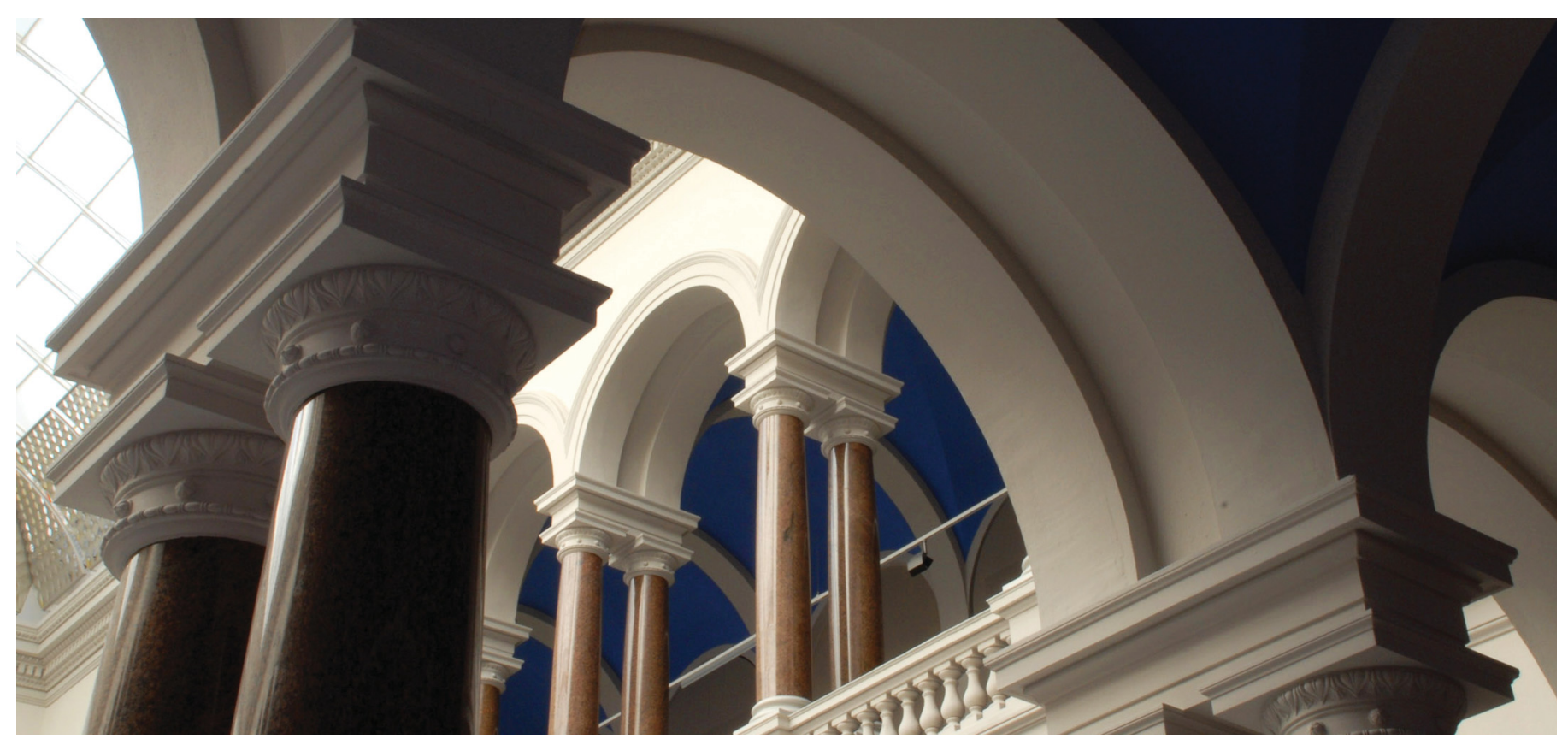

This is a post-peer-review, pre-copyedit version of an article published in Acta Geotechnica. The final authenticated version is available online at: http://dx.doi.org/10.1007/s11440-019-00792-z.

Aubram, D. (2019). Explicitly coupled consolidation analysis using piecewise constant pressure. Acta Geotechnica. https://doi.org/10.1007/s11440-019-00792-z 


\title{
Explicitly coupled consolidation analysis using piecewise constant pressure
}

\author{
Daniel Aubram
}

Received: date / Accepted: date

\begin{abstract}
The paper describes an explicit coupling procedure for efficient consolidation analysis. Each time step is divided into a flow step followed by a drained mechanical step. The flow step keeps the total mean stress increment fixed and solves a diffusion problem based on piecewise constant pressure data. The procedure can be added to purely mechanical finite element codes and does not require a fully coupled element type. Details on discretization and implementation are provided as well as results of numerical tests.
\end{abstract}

Keywords Consolidation · Coupled · Explicit · Flow · Darcy · Diffusion

\section{Introduction}

Consolidation of saturated soil is characterized by the interaction of the solid displacement field with the flow field of the pore fluid. The widely recognized numerical approach for consolidation analysis utilizes the finite element method and a fully coupled procedure which solves the governing equations simultaneously at every time step $[11,16]$.

Fully coupled solution procedures often require enormous software development and can be computationally expensive. This is particularly true for multi-physics and multi-material problems where consolidation might be only one physical aspect of the problem, and only locally, in small regions of the computational domain $[1,3]$. In such situations, and others, less rigorous coupling procedures can be more efficient $[15,12,10]$.

This paper is concerned with the development of an explicit (i.e. sequential noniterative) coupling procedure for consolidation analysis based on a fixed-stress split of the governing equations $[12,10]$. The total mean stress increment is kept constant when solving the flow problem associated with the continuity equation and Darcy's law. Thereafter, the drained mechanical problem is solved by imposing the pore

D. Aubram

Technische Universität Berlin (TU Berlin), Chair of Soil Mechanics and Geotechnical Engineering, Secr. TIB1-B7, Gustav-Meyer-Allee 25, 13355 Berlin, Germany

E-mail: daniel.aubram@tu-berlin.de 
pressure gradient as a body force density. The initial pore pressure and effective stress fields are determined during an undrained mechanical analysis at the beginning of the calculation.

Our numerical implementation is intended as a simple approach to add the consolidation analysis capability to a purely mechanical finite element code without a fully coupled element type. The mechanical steps use standard element technology [4] whereas the flow step employs the five-point stencil in a finite difference discretization [8]. The pore pressure gradient is approximated using central differences.

\section{Method}

\subsection{Governing equations}

The following section derives the basic equations of three-dimensional consolidation theory and is partially based on derivations in $[14,16]$. We use the sign convention of general mechanics.

By neglecting inertia forces and body forces, e.g. due to gravity, balance of linear momentum of a saturated porous medium can be written

$$
\operatorname{div} \boldsymbol{\sigma}=\mathbf{0} \quad \text { or } \quad \operatorname{div} \boldsymbol{\sigma}^{\prime}=\nabla p,
$$

where $\boldsymbol{\sigma}$ is the total stress, $\boldsymbol{\sigma}^{\prime}$ is the effective stress, $p$ is the pore fluid pressure, and $\nabla$ is the del operator. The crucial observation that motivates our method is that the pressure gradient is equivalent to a body force density if the total stress in (1) is replaced with the effective stress; cf. Appendix A for a derivation.

Eq. (1) involves Terzaghi's principle of effective stress, whose rate form states that

$$
\dot{\boldsymbol{\sigma}}=\dot{\boldsymbol{\sigma}}^{\prime}-\dot{p} \boldsymbol{I}, \quad \text { leading to } \quad \dot{P}=\dot{p}^{\prime}+\dot{p},
$$

where $\boldsymbol{I}$ is the second-order unit tensor, $p^{\prime}=-\frac{1}{3} \operatorname{tr} \boldsymbol{\sigma}^{\prime}$ is the mean effective stress, $P=-\frac{1}{3} \operatorname{tr} \boldsymbol{\sigma}$ is the mean total stress, $\operatorname{tr} \boldsymbol{s}=\boldsymbol{I}: \boldsymbol{s}$ is the trace of any second-order tensor $\boldsymbol{s}$, : denotes double contraction, and a superposed dot denotes the material time derivative.

Under the assumption of infinitesimal strains, a generic constitutive equation for the rate of effective stress can be defined through

$$
\dot{\sigma}^{\prime}=C: \dot{\varepsilon},
$$

where $\boldsymbol{C}$ is a fourth-order material tangent tensor representing the stiffness of the drained porous medium, $\dot{\boldsymbol{\varepsilon}}=\frac{1}{2}\left(\nabla \dot{\boldsymbol{u}}+(\nabla \dot{\boldsymbol{u}})^{\mathrm{T}}\right)$ is the rate of strain, $\boldsymbol{u}$ is the displacement of the solid phase, and a superscript $\mathrm{T}$ denotes the transpose of a second-order tensor. The material tangent is generally a function of the effective stress state and other history variables.

It should be noticed that the trace of the strain rate, $\operatorname{tr} \dot{\varepsilon}=\boldsymbol{I}: \dot{\varepsilon}=\dot{\varepsilon}_{\mathrm{v}}=\dot{V} /\left.V\right|_{M_{\mathrm{s}}}$, describes the rate of total change of an elementary volume $V$ of the porous medium by keeping the solid mass $M_{\mathrm{s}}$ constant. The bulk modulus of the drained porous medium, $K$, is then defined by

$$
\dot{p}^{\prime}=-K \dot{\varepsilon}_{\mathrm{v}}, \quad \text { with } \quad K=\frac{1}{9} \boldsymbol{I}: \boldsymbol{C}: \boldsymbol{I} .
$$


Continuity requires that the total volume change equals the sum of a volume change by compression of the pore fluid and a volume change as a result of a net outflow. Conceptually,

$$
\dot{\varepsilon}_{\mathrm{v}}=\dot{\varepsilon}_{\mathrm{v} 1}+\dot{\varepsilon}_{\mathrm{v} 2}
$$

The solid material of the porous medium is assumed incompressible. Concerning the compression of the pore fluid,

$$
\dot{\varepsilon}_{\mathrm{v} 1}=-\frac{n}{K_{\mathrm{f}}} \dot{p}
$$

where $n$ is the porosity and $K_{\mathrm{f}}$ the fluid bulk modulus.

The net outflow rate under drained conditions is described by the divergence of the specific discharge $\boldsymbol{q}$. The specific discharge is modeled by Darcy's law, leading to

$$
\dot{\varepsilon}_{\mathrm{v} 2}=-\operatorname{div} \boldsymbol{q}=\operatorname{div}\left(\frac{k}{\gamma_{\mathrm{f}}} \nabla p\right)=\frac{k}{\gamma_{\mathrm{f}}} \nabla^{2} p,
$$

where $k$ is the hydraulic conductivity, which is assumed isotropic, $\gamma_{\mathrm{f}}$ is the unit weight of the pore fluid, and $\nabla^{2}=\nabla \cdot \nabla$ is the Laplace operator. Substitution of (6) and (7) into (5) then gives the storage equation

$$
\dot{\varepsilon}_{\mathrm{v}}=-\frac{n}{K_{\mathrm{f}}} \dot{p}+\frac{k}{\gamma_{\mathrm{f}}} \nabla^{2} p
$$

Rewriting (8) by using (2) and (4) yields

$$
\dot{p}=\frac{S}{K} \dot{P}+c_{p} \nabla^{2} p
$$

with

$$
\frac{1}{S}=\frac{1}{K}+\frac{n}{K_{\mathrm{f}}} \quad \text { and } \quad c_{p}=\frac{S k}{\gamma_{\mathrm{f}}} .
$$

$c_{p}$ is called the consolidation coefficient.

Equations (1) and (9) in conjunction with (2) and (3) constitute a coupled set of equations for the unknowns $\boldsymbol{u}$ and $p$. Coupling occurs because the total stress at a point generally changes as the porous medium deforms during consolidation.

We note that under locally undrained conditions, $\dot{\varepsilon}_{\mathrm{v}}=\dot{\varepsilon}_{\mathrm{v} 1}$ resp. $\operatorname{div} \boldsymbol{q}=0$. In this case, (6) might be directly substituted into (2), resulting in the constitutive equation

$$
\dot{\boldsymbol{\sigma}}=\left(\boldsymbol{C}+\frac{K_{\mathrm{f}}}{n} \boldsymbol{I} \otimes \boldsymbol{I}\right): \dot{\varepsilon}_{\mathrm{u}}=\boldsymbol{C}_{\mathrm{u}}: \dot{\varepsilon}_{\mathrm{u}}
$$

for the total stress by using (3). $\boldsymbol{C}_{\mathrm{u}}$ represents the undrained material tangent tensor and $\dot{\varepsilon}_{\mathrm{u}}$ the rate of strain of the undrained solid-fluid mixture. The tensor product $\boldsymbol{I} \otimes \boldsymbol{I}$ defines a fourth-order unit tensor that maps a second-order tensor $s$ onto its spherical part $(\operatorname{tr} \boldsymbol{s}) \boldsymbol{I}=\boldsymbol{I} \otimes \boldsymbol{I}: \boldsymbol{s}$. 
2.2 Initial and boundary conditions

Consolidation generally is a three-dimensional initial boundary value problem. However, in the present study we consider only consolidation problems which can be approximated by plane strain and plane flow conditions, so that the computational domain $\mathcal{B} \in \mathbb{R}^{2}$ is two-dimensional. A particular consolidation problem is the problem of determining the solid displacement $\boldsymbol{u}$ and pore pressure $p$ on $\mathcal{B}$ for every time $t \in[0, T]$ by solving (1) and (9) subject to the following initial conditions and boundary conditions $(\mathrm{BC})$ specified on the boundary $\partial \mathcal{B}$ :

$$
\begin{aligned}
\left.\boldsymbol{\sigma}^{\prime}\right|_{t=0}=\boldsymbol{\sigma}_{0}^{\prime}, & \left.\boldsymbol{u}\right|_{t=0}=\boldsymbol{u}_{0}, \quad \text { and }\left.p\right|_{t=0}=p_{0} \\
\boldsymbol{u}=\overline{\boldsymbol{u}} & \text { on } \partial_{\boldsymbol{u}} \mathcal{B}(\text { displacement BC) } \\
\boldsymbol{\sigma}^{\prime} \cdot \boldsymbol{n}=\overline{\boldsymbol{t}} & \text { on } \partial_{\boldsymbol{t}} \mathcal{B}(\text { traction BC) } \\
p=\bar{p} & \text { on } \partial_{p} \mathcal{B}(\text { pore pressure BC) } \\
\boldsymbol{q} \cdot \boldsymbol{n}=\bar{q}_{n} & \text { on } \partial_{q} \mathcal{B}(\text { flux BC) }
\end{aligned}
$$

Eqs. (12) formalize the initial conditions, (13) and (14) are the boundary conditions for the mechanical sub-problem, and (15) and (16) are the boundary conditions for the flow sub-problem. Here $\boldsymbol{n}$ are the unit outward normals on $\partial \mathcal{B}$, and $\overline{\boldsymbol{u}}, \overline{\boldsymbol{t}}, \bar{p}$ and $\bar{q}_{n}$ are prescribed values of displacement, traction, pore pressure and normal flux, respectively. Moreover, the total boundary is the union $\partial_{\boldsymbol{u}} \mathcal{B} \cup \partial_{\boldsymbol{t}} \mathcal{B}=\partial_{p} \mathcal{B} \cup \partial_{q} \mathcal{B}=\partial \mathcal{B}$, and the intersection of each pair is the empty set.

\subsection{Discretization}

Although different sequential coupling procedures are available in the literature, superior stability and convergence properties are reported for the fixed-stress split [10]. Moreover, when the fixed-stress split is implemented into a purely mechanical finite element code, as done in the present research, the displacements of the drained porous medium and the effective stress are direct output, while other sequential schemes would require post-processing of the results.

Let the time interval $[0, T] \subset \mathbb{R}$ be partitioned into a sequence $\left(t_{0}=0, \ldots, t_{n+1}=\right.$ $\left.t_{n}+\Delta t, \ldots, T\right)$ of discrete time stations, with constant time increment $\Delta t$. Given a solution at time $t=t_{n}$, the fixed-stress split divides each time increment into a flow step followed by a drained mechanical step to obtain the solution at $t=t_{n+1}$ :

$$
\left(\begin{array}{l}
\boldsymbol{u}_{n} \\
p_{n}
\end{array}\right) \stackrel{\text { flow }}{\longrightarrow}\left(\begin{array}{c}
\boldsymbol{u}_{n+1}^{*} \\
p_{n+1}
\end{array}\right) \stackrel{\text { mech. }}{\longrightarrow}\left(\begin{array}{c}
\boldsymbol{u}_{n+1} \\
p_{n+1}
\end{array}\right) \text {. }
$$

$\boldsymbol{u}_{n+1}^{*}$ is a predictor of the updated displacement field caused by the total volume change $\Delta \varepsilon_{\mathrm{v}}$ during the flow step. Calculation starts with a locally undrained mechanical step to initialize the pore pressure and effective stress fields.

In the mechanical step, we use standard finite element technology to solve (1) subject to (12)-(14) by taking the rate constitutive equation (3) resp. (11) into account $[4,2]$. Quadrilateral elements with one-point integration and hourglass control prevent volumetric locking in cases where large $K_{\mathrm{f}}$ cause nearly incompressible behavior of the locally undrained solid-fluid mixture. 
The mechanical step imposes the pore pressure gradient $\nabla p$ as a body force density and results in nodal incremental displacements $\Delta \boldsymbol{u}$ used to compute an incremental strain $\Delta \varepsilon$ at the integration points of finite elements. The effective stress during the drained step is then updated according to

$$
\boldsymbol{\sigma}_{n+1}^{\prime}=\boldsymbol{\sigma}_{n}^{\prime}+\Delta \boldsymbol{\sigma}^{\prime}(\Delta \varepsilon)
$$

where $\Delta \boldsymbol{\sigma}^{\prime}=\int_{t_{n}}^{t_{n+1}} \dot{\boldsymbol{\sigma}}^{\prime} \mathrm{d} t$ and $\dot{\boldsymbol{\sigma}}^{\prime}$ is defined through (3). Similar holds for the total stress update during the undrained initialization step which employs (11).

The mechanical step and the pore pressure increment determined in the previous time step result in a total mean stress increment $\Delta P=\Delta p^{\prime}+\Delta p$. The total mean stress increment is kept constant during the subsequent flow step, which solves the modified storage equation (9) subject to (15) and (16) by using a forward difference approximation to the time derivative:

$$
\frac{p_{n+1}-p_{n}}{\Delta t}=\frac{S}{K} \frac{\Delta P}{\Delta t}+c_{p} \nabla^{2} p_{n}
$$

The spatial discretization of the last term in (19) is crucial in the solution of the associated diffusion problem. Let us assume piecewise constant, element-centered pressure data on a uniform grid. Such a grid is readily available if the mechanical step approximates the computational domain by a structured quadrilateral mesh $\mathcal{B} \approx \bigcup_{e=1}^{n_{\mathrm{el}}} \Omega_{e}$ consisting of $n_{\mathrm{el}}$ finite elements $\Omega_{e} \subset \mathbb{R}^{2}$. Accordingly, each element $e$ consists of four nodes $i \in\{1,2,3,4\}$ in counterclockwise order along the perimeter, and four edges $\Gamma_{e, i}$ starting at node $i$, with $\partial \Omega_{e} \approx \bigcup_{i} \Gamma_{e, i}$. Moreover, the element centroids form the points of the finite difference grid used for the flow step, and the data associated with those points is the element average of the corresponding finite element data.

A central difference approximation to the second space derivatives results in a five-point finite difference stencil [8], so that (19) becomes

$$
p_{n+1}=p_{n}+\frac{S}{K} \Delta P+\frac{c_{p} \Delta t}{h^{2}}\left(\sum_{i} p_{\text {adj }(e, i)}-4 p_{e}\right)
$$

$h$ is the characteristic element length, $p_{e}$ is the average value of $p_{n}$ in element $e$, and $\operatorname{adj}(e, i)$ denotes the adjacent element of $e$ sharing the common edge $\Gamma_{e, i}$. It can be shown $[14,8]$ that the explicit algorithm (20) is stable provided that $\Delta t<h^{2} /\left(4 c_{p}\right)$.

Once the pore pressure has been updated, its gradient needed to solve (1) can be computed by using a central difference approximation [8]. For example, the component of the pore pressure gradient in element $e$ in direction normal to the edge $\Gamma_{e, i}$ is given by

$$
\nabla p_{\perp \Gamma_{e, i}}=\frac{1}{2 h}\left(p_{\operatorname{adj}(e, i)}-p_{\operatorname{adj}(e, i+2)}\right)
$$

At boundaries, evaluation of (20) and (21), and imposing the flow boundary conditions (15) and (16) is facilitated by adding "ghost" elements [8], i.e. rows of (nonexistent) elements outside of the computational domain. 
2.4 Numerical implementation

We implemented the explicit coupling procedure into a purely mechanical finite element code. The intention was to add the capability of consolidation analysis by keeping programming of user interfaces to a minimum. The mechanical steps do not pose a challenge; setting the boundary conditions (13) and (14) is the same for drained and undrained mechanical analyses.

A convenient implementation of the flow step and pressure gradient calculation defines a set of common variables that can be accessed by the user across different program units. Among these common variables are the pore fluid pressure, the pressure gradient, and the list of adjacent elements for all elements in the mesh. The latter is necessary to implement (20) but is not normally known in a standard finite element analysis, so we provide a method for determining it in Appendix B.

To implement the Dirichlet boundary condition (15) and Neumann boundary condition (16), we proceed as follows. For simplicity, we assume

$$
\bar{p}=0 \text { on } \partial_{p} \mathcal{B} \quad \text { and } \quad \bar{q}_{n}=0 \text { on } \partial_{q} \mathcal{B} .
$$

A flag is stored for each element in an array ppres_bc of length $n_{\mathrm{el}}$ which indicates whether a Dirichlet boundary condition is set for that element. If this is the case, the element is regarded fully drained and consolidated and hence the element pore pressure, $p_{n+1}$, and pore pressure increment are set to zero.

All elements at boundaries of the underlying finite element mesh for which no Dirichlet boundary condition is specified are treated as Neumann boundary elements. The boundary condition (16) can be easily handled when using the five-point stencil, (20). First, we note that $\boldsymbol{q} \cdot \boldsymbol{n}=\bar{q}_{n}=0$ implies that the component of the pore pressure gradient $\nabla p$ normal to the boundary vanishes. For the assumed uniform square mesh, let $\Gamma_{e, i}$ be an edge of element $e$ aligned with that boundary. Then, setting (21) to zero results in

$$
p_{\operatorname{adj}(e, i)}=p_{\operatorname{adj}(e, i+2)} .
$$

Here $p_{\operatorname{adj}(e, i)}$ is the pressure in the "ghost" element adjacent to $\Gamma_{e, i}$, and $p_{\operatorname{adj}(e, i+2)}$ is the pressure in the element adjacent to the opposite edge $\Gamma_{e, i+2}$. In order to implement the Neumann boundary condition, the identity (23) is substituted into (20) whenever there is no "real" adjacent element $\operatorname{adj}(e, i)$.

The overall explicit coupling procedure can be summarized as follows:

1. Specify initial and boundary conditions

2. Initialize $p, \boldsymbol{\sigma}^{\prime}$ through undrained analysis

3. Solve pore pressure diffusion step:

(a) Update $p$ using (20) and $\Delta P$

(b) Handle BCs (15) and (16); cf. Sect. 2.4

(c) Determine $\nabla p$

4. Solve drained mechanical step:

(a) Determine $\Delta \boldsymbol{u}$ from (1), (13) and (14)

(b) Update $\boldsymbol{\sigma}^{\prime}$ using (18) and (3)

(c) Compute $\Delta P=\Delta p^{\prime}+\Delta p$

5. Update time $t_{n} \leftarrow t_{n+1}$; go to 3 
Table 1 Material parameters used in calculations

\begin{tabular}{lll}
\hline Description & Symbol & Value \\
\hline Young's modulus & $E$ & $1.5 \times 10^{4} \mathrm{kN} \mathrm{m}^{-2}$ \\
Poisson's ratio & $\nu$ & 0.25 \\
porosity & $n$ & 0.4 \\
hydraulic conductivity & $k$ & $1 \times 10^{-4} \mathrm{~m} \mathrm{~s}^{-1}$ \\
fluid bulk modulus & $K_{\mathrm{f}}$ & $2 \times 10^{6} \mathrm{kN} \mathrm{m}^{-2}$ \\
fluid unit weight & $\gamma_{\mathrm{f}}$ & $10 \mathrm{kN} \mathrm{m}^{-3}$ \\
\hline
\end{tabular}

\section{Results}

Two example problems are analyzed in order to test our method. In all calculations we assume isotropic linear elastic behavior of the porous material. Therefore, the material tangent in (3) can be represented by Young's modulus $E$ and Poisson's ratio $\nu$. The complete set of material parameters is listed in Tab. 1.

All calculations were run using a uniform mesh made up of quadrilateral elements; cf. Sect. 2.3. Results are plotted using the dimensionless consolidation time $T_{\mathrm{c}}=$ $c_{p}^{*} t / H^{2}$ and degree of consolidation $U_{\mathrm{c}}=\left(u_{z}-u_{z 0}\right) /\left(u_{z \infty}-u_{z 0}\right)$, where $u_{z 0}$ and $u_{z \infty}$ denote the vertical displacement of a point at ground surface at times $t=0$ and $t \rightarrow \infty$, respectively. Note that $c_{p}^{*}$ is an adjusted consolidation coefficient which uses the constrained modulus $E_{\mathrm{s}}=E(1-\nu) /((1+\nu)(1-2 \nu))$ instead of the drained bulk modulus $K$; cf. (10).

\subsection{One-dimensional consolidation}

Terzaghi derived an analytical solution for one-dimensional consolidation under a constant load [14]. The computational model used for back-calculation consists of 200 finite elements arranged in a single column with total height $H=1.0 \mathrm{~m}$. The lower boundary is fixed in both directions whereas nodes at the vertical boundaries are fixed in horizontal direction. At the upper boundary, $\bar{p}=0$, i.e. the porous medium is free to drain under a pressure load $10 \mathrm{kN} \mathrm{m}^{-2}$. Zero normal flux, $\bar{q}_{n}=0$, is prescribed at all other boundaries.

The simulated distribution of the pore pressure at different dimensionless consolidation times $T_{\mathrm{c}}$ agrees well with the analytical solution (Fig. 1). The analytical solution $u_{z, \infty}=-10 / E_{\mathrm{s}}=-5.55 \times 10^{-4} \mathrm{~m}$ is matched with an error of less than $0.1 \%$.

\subsection{Two-dimensional consolidation}

The second example addresses two-dimensional consolidation of a finite soil layer subject to a vertical load on a part of the surface. The computational model shown in Fig. 2 above has a height of $H=4 \mathrm{~m}$, a width of $16 \mathrm{~m}$, and takes advantage of symmetry. The left, right, and lower boundaries are impermeable (zero normal flux) and fixed in normal direction. At the ground surface, $\bar{p}=0$ is imposed together with a left-justified pressure load of $10 \mathrm{kN} \mathrm{m}^{-2}$ over a width of $4 \mathrm{~m}$. 


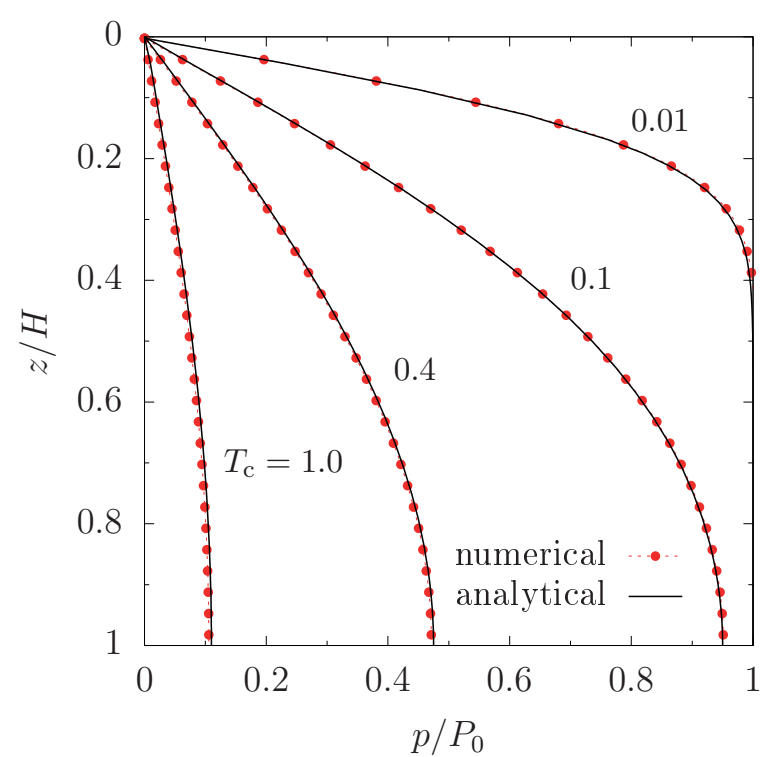

Fig. 1 Distribution of normalized pore pressure at different dimensionless consolidation times for the 1D consolidation example
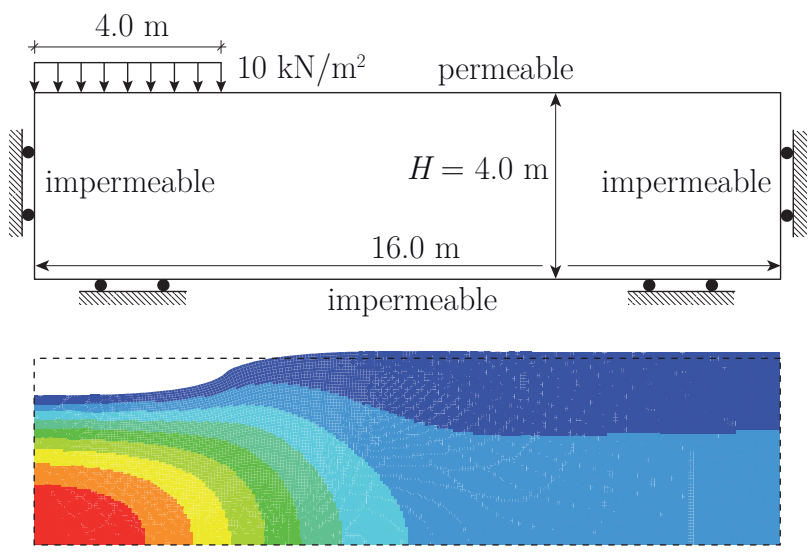

$p$ in $\mathrm{kN} / \mathrm{m}^{2}\left(T_{\mathrm{c}}=0.1\right)$

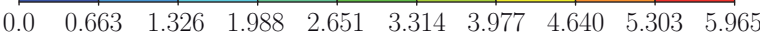

Fig. 2 Two-dimensional consolidation: model geometry and boundary conditions (above), deformed model and pore pressure distribution at $T_{\mathrm{c}}=0.1$ (below); displacements are scaled so that maximum displacement displays as $5 \%$ of the model width 


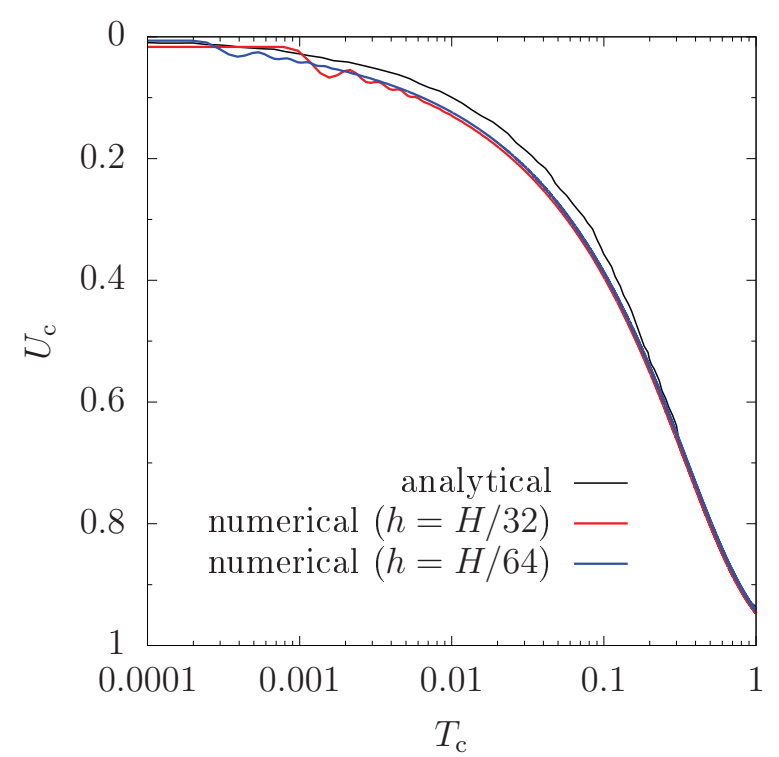

Fig. 3 Degree of consolidation for the upper left corner as a function of dimensionless consolidation time for the $2 \mathrm{D}$ consolidation example

The calculated deformations of the ground surface and the pore pressure distribution at $T_{\mathrm{c}}=0.1$ shown in Fig. 2 below are reasonable. The simulated time history of $U_{\mathrm{c}}$ for the upper left corner converges to the analytical solution of Booker [6] as the element size $h$ is reduced (Fig. 3).

\section{Conclusions and outlook}

In this paper we have developed, implemented, and tested an explicit coupling procedure for consolidation analysis based on the fixed-stress split of the governing equations. Our numerical implementation is currently restricted to uniform structured meshes. This, in fact, has enabled solution of the pore pressure diffusion problem by using the classical five-point finite difference stencil.

The proposed method is not intended to save computing time compared to fully coupled schemes when solving problems where soil consolidation is the exclusive physical aspect. Explicit coupling instead has inherent advantages from the viewpoint of customization, modularity of code, and reusing existing software. Therefore, the strength of the proposed method will become apparent when the problem under consideration has other physical aspects (multi-physics problem), or when consolidation is a localized phenomenon in a multi-material problem.

Future research will be concerned with accuracy assessment and the extension of the procedure to general unstructured meshes and multi-material situations with heterogeneous diffusion coefficients. Methods that can handle such problems are based on, for example, support-operators resp. mimetic finite differences [13, 7]. These are, however, much more complex than the method presented here. 


\section{Appendix A}

By taking into account a non-zero body force per unit volume, $\boldsymbol{b}$, (1) reads

$$
\operatorname{div} \boldsymbol{\sigma}+\boldsymbol{b}=\operatorname{div}\left(\boldsymbol{\sigma}^{\prime}-p \boldsymbol{I}\right)+\boldsymbol{b}=\mathbf{0} .
$$

For the second term, tensor calculus [9] yields

$$
\begin{array}{r}
\operatorname{div}(p \boldsymbol{I})=\frac{\partial}{\partial x_{k}}\left(p \delta_{i j} \boldsymbol{e}_{i} \otimes \boldsymbol{e}_{j}\right) \cdot \boldsymbol{e}_{k}=\frac{\partial p}{\partial x_{k}} \delta_{i j} \boldsymbol{e}_{i}\left(\boldsymbol{e}_{j} \cdot \boldsymbol{e}_{k}\right) \\
=\frac{\partial p}{\partial x_{k}} \boldsymbol{e}_{i} \delta_{i j} \delta_{j k}=\frac{\partial p}{\partial x_{i}} \boldsymbol{e}_{i}=\nabla p
\end{array}
$$

where $i, j, k \in\{1,2,3\}$, the $x_{i}$ are Cartesian coordinates, $\boldsymbol{e}_{i}$ denote the standard basis vectors, $\delta_{i j}=\boldsymbol{e}_{i} \cdot \boldsymbol{e}_{j}$ is the Kronecker delta, and $\boldsymbol{I}=\delta_{i j} \boldsymbol{e}_{i} \otimes \boldsymbol{e}_{j}$, where $\otimes$ denotes the tensor product. Substitution and rearranging terms then results in

$$
\operatorname{div} \boldsymbol{\sigma}^{\prime}=\nabla p-\boldsymbol{b}
$$

This shows that $-\nabla p$ is equivalent to a body force density if the total stress in (1) is replaced with the effective stress.

\section{Appendix B}

Implementation of (20) requires the list of elements adjacent to each element sharing a common edge. An efficient algorithm for determining this list has been suggested in [5], which is reproduced here for the sake of completeness.

Let nel be the number of elements and nnp the number of nodal points in a finite element mesh composed of four-node quadrilaterals only. Given data on the mesh is the connectivity matrix, cnct, which assigns a global node number $n=\operatorname{cnct}(e, i)$ to the element number e and local node number $i=1 \ldots 4$.

The list of adjacent elements is stored in an array eadj(e,i). To efficiently generate it, a list of elements attached to each node is generated in an intermediate step. The latter is stored in a one-dimensional array $1 \mathrm{cn}$ of length $4 * n e l$ and requires an array of pointers, lcn_pt, and the number of elements connected to the nodes, lcn_num, which both have a length of nnp.

After counting the number of elements connected to each node by

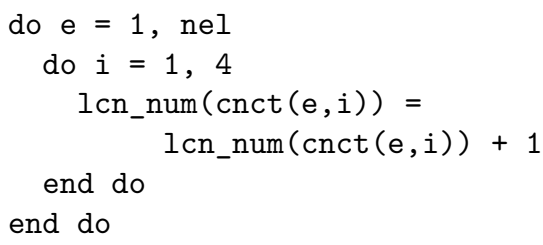

the pointers are calculated. The pointers are defined such that $l \mathrm{cn}\left(\mathrm{lcn}_{-} \mathrm{pt}(\mathrm{n})+\mathrm{k}\right)$ is the $\mathrm{k}$-th element connected to node $\mathrm{n}$ : 


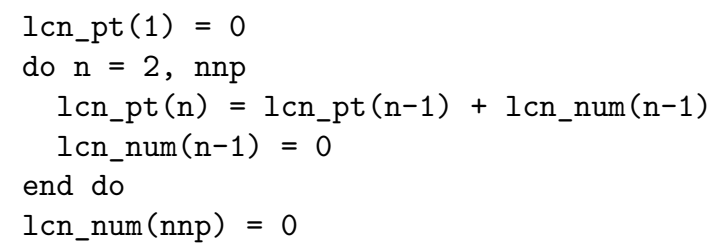

Note that each $1 \mathrm{cn} \_$num(n) is set back to zero to use it as a counter during the filling of $1 \mathrm{cn}$ :

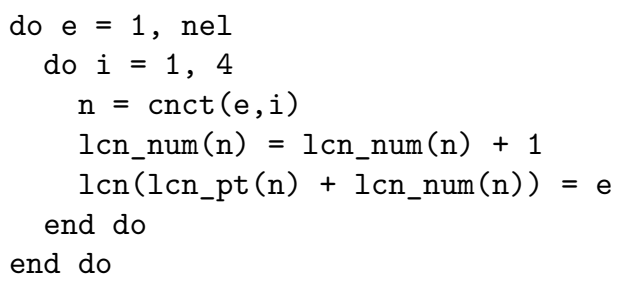

An edge $i$ of element $e$ is defined by node $i$ and the node counterclockwise from it, next_nd $(i)=i+1$, with $i+1=1$ for $i=4$. The edge is shared by only two elements, the current element e and the adjacent element, eadj (e,i). A search on the lists of elements connected to the two nodes of the edge is carried out in order to determine the two elements in common. The element which is not the current element is the adjacent element.

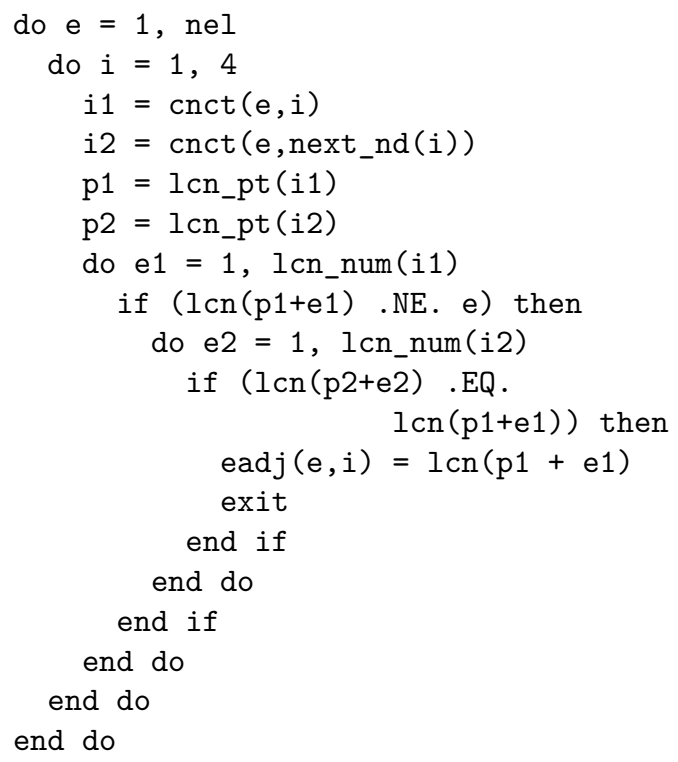

It should be noted that eadj is initialized with zeros. Therefore, once the list of adjacent elements has been determined, a zero at eadj (e,i) indicates that edge $i$ of element $\mathrm{e}$ is aligned with a mesh boundary. 


\section{References}

1. Aubram D (2016) Homogeneous equilibrium model for geomechanical multimaterial flow with compressible constituents. Journal of Non-Newtonian Fluid Mechanics 232:88-101

2. Aubram D (2017) Notes on rate equations in nonlinear continuum mechanics. arXiv e-prints 1709.10048 [physics.class-ph]

3. Aubram D, Rackwitz F, Savidis SA (2017) Contribution to the non-Lagrangian formulation of geotechnical and geomechanical processes. In: Triantafyllidis $\mathrm{T}$ (ed) Lecture Notes in Applied and Computational Mechanics, vol 82, Springer International Publishing

4. Belytschko T, Liu WK, Moran B, Elkhodary KI (2014) Nonlinear Finite Elements for Continua and Structures, 2nd edn. John Wiley \& Sons, Chichester, $\mathrm{UK}$

5. Benson DJ (2008) Momentum advection on unstructured staggered quadrilateral meshes. International Journal for Numerical Methods in Engineering 75:15491580

6. Booker JR (1974) The consolidation of a finite layer subject to surface loading. International Journal of Solids and Structures 10:1053-1065

7. Breil J, Maire PH (2007) A cell-centered diffusion scheme on two-dimensional unstructured meshes. Journal of Computational Physics 224:785-823

8. Hirsch C (2007) Numerical Computation of Internal and External Flows, vol 1, 2nd edn. Butterworth-Heinemann, Burlington, MA, USA

9. Holzapfel GA (2000) Nonlinear Solid Mechanics - A Continuum Approach for Engineering, John Wiley \& Sons, Chichester, UK

10. Kim J, Tchelepi HA, Juanes R (2011) Stability, accuracy and efficiency of sequential methods for coupled flow and geomechanics. SPE Journal 16(2):249-262, document ID: SPE-119084-PA

11. Lewis RW, Schrefler BA (1998) The Finite Element Method in the Static and Dynamic Deformation and Consolidation of Porous Media, 2nd edn. John Wiley \& Sons, Ltd., Chichester

12. Settari A, Walters DA (2001) Advances in coupled geomechanical and reservoir modeling with applications to reservoir compaction. SPE Journal 6(3):334-342, document ID: SPE-74142-PA

13. Shashkov M, Steinberg S (1996) Solving diffusion equations with rough coefficients in rough grids. Journal of Computational Physics 129(2):383-405

14. Verruijt A (1995) Computational Geomechanics, Theory and Applications of Transport in Porous Media, vol 7. Springer Science+Business Media, Dordrecht, The Netherlands

15. Zienkiewicz OC, Paul DK, Chan AHC (1988) Unconditionally stable staggered solution procedure for soil-pore fluid interaction problems. International Journal for Numerical Methods in Engineering 26(5):1039-1055

16. Zienkiewicz OC, Chan AHC, Pastor M, Schrefler BA, Shiomi T (1999) Computational Geomechanics with Special Reference to Earthquake Engineering. John Wiley \& Sons, Chichester, UK 